\title{
SIMPLY CONNECTED SPIN MANIFOLDS WITH POSITIVE SCALAR CURVATURE
}

\author{
TETSURO MIYAZAKI
}

\begin{abstract}
Let $l_{n}$, be equal to 2 or 4 according as $n$ is congruent $\bmod 8$ to zero or not. If the $\hat{A}$-genus of a compact simply connected spin manifold of dimension $n \geqslant 5$ vanishes, then the connected sum of $l_{n}$ copies of the manifold admits a metric of positive scalar curvature. This supports a conjecture of Gromov and Lawson.
\end{abstract}

Introduction. In relation to the works of Kazdan and Warner and others (see [2]), it is an important problem to describe the condition for a given manifold to admit a metric of positive scalar curvature.

Lichnerowicz' theorem [6] states that the vanishing of the $\hat{A}$-genus is one of the necessary conditions for spin manifolds to have such metrics. Moreover, Hitchin [5] proved a vanishing theorem for the KO-characteristic number $\hat{\mathscr{A}}: \Omega_{*}^{\mathrm{Spin}} \rightarrow \mathrm{KO}$ (pt.), which essentially coincides with the $\hat{A}$-genus in dimensions congruent to zero mod 4 .

On the other hand, a cobordism-theoretic approach was achieved in one of the papers of Gromov and Lawson [4]. They showed that every compact simply connected manifold which is not spin and whose dimension is not less than 5 carries a metric of positive scalar curvature. Also they showed that if the $\hat{A}$-genus of a compact simply connected manifold of dimension not less than 5 vanishes, the connected sum of some copies of the manifold carries a metric of positive scalar curvature. They conjectured that for a compact simply connected manifold of dimension not less than 5 , the KO-characteristic number $\hat{\mathscr{A}}$ is the complete obstruction to the existence of a metric of positive scalar curvature.

Let us put it in another way. Let $P \subset \Omega_{*}^{\text {Spin }}$ be the ideal consisting of the set of classes containing representatives with positive scalar curvature. We consider the homomorphism

$$
\Pi: \Omega_{*}^{\text {Spin }} \rightarrow \Omega_{*}^{\text {Spin }} / P .
$$

Gromov and Lawson proved that a simply connected spin manifold which is spin cobordant to a spin manifold of positive scalar curvature also admits a metric of positive scalar curvature. Therefore, the conjecture of Gromov and Lawson means that $\operatorname{ker} \hat{\mathscr{A}}$ coincides with $P$ and, as a result, $\Pi$ and $\Omega_{*}^{\text {Spin }} / P$ coincide with $\hat{\mathscr{A}}$ and $\mathrm{KO}_{*}(\mathrm{pt}$.), respectively. They proved that $\Pi \otimes Q$ is exactly the $\hat{A}$-genus.

Received by the editors June 4, 1984.

1980 Mathematics Subject Classification. Primary 57D75, 53C20.

Key words and phrases. Positive scalar curvature, spin cobordism.

(1985 American Mathematical Society $0002-9939 / 85 \$ 1.00+\$ .25$ per page 
In this paper, we show a more refined result concerning this conjecture. Note that the theorem of Hitchin guarantees that $P$ is contained in ker $\hat{\mathscr{A}}$. What we want is its converse. Let $l_{k}$ be equal to 4 or 2 according as $k$ is congruent mod 8 to zero or not. We denote $\operatorname{ker} \hat{\mathscr{A}} \cap \Omega_{k}^{\text {Spin }}$ and $P \cap \Omega_{k}^{\text {Spin }}$ by $\operatorname{ker} \hat{\mathscr{A}}_{k}$ and $P_{k}$ respectively. Our result is the following.

TheOREM. $l_{k} \operatorname{ker} \hat{\mathscr{A}}_{k}$ is contained in $P_{k}$. In particular, let $M$ be a compact simply connected manifold of dimension $k \geqslant 5$ whose $\hat{A}$-genus vanishes. Then the connected sum of $l_{k}$ copies of $M$ admits a metric of positive scalar curvature.

Note that this implies that the conjecture is true mod any odd number $p$, i.e. $\Pi \otimes \mathbf{Z}_{p}$ coincides with $\hat{\mathscr{A}} \otimes \mathbf{Z}_{p}$.

The conjecture of Gromov and Lawson has a close relation to the following problem. If some multiple $M \# \cdots \# M$ carries a metric of positive scalar curvature, then does $M$ itself carry such a metric? In general, the answer is no even in the case of simply connected spin manifolds. In fact, there exists a 9-dimensional simply connected spin manifold $M$ such that the KO-characteristic number $\hat{\mathscr{A}}$ does not vanish and such that $M \# M$ carries positive scalar curvature (it is cobordant to $S^{9}$ ). However, our Theorem asserts that the answer is yes for odd numbers.

COROLlARY. Let $M$ be a compact simply connected spin manifold such that the odd multiple $M \# \cdots \#$ carries a metric of positive scalar curvature. Then $M$ itself carries such a metric.

REMARK. One can show that each class of $\operatorname{ker} \hat{\mathscr{A}}_{k}(k \leqslant 12)$ or $\operatorname{ker} \hat{\mathscr{A}}_{k} / \operatorname{Tor}(k \leqslant 20)$ is represented by a spin manifold of positive scalar curvature using $\mathbf{H P}^{4}$ and manifolds in our proof and in [7]. This means that the conjecture is true in low dimensions.

Concluding this Introduction, the author wants to propose the following problem.

Problem. Can each class in the kernel of the map

$$
\hat{\mathscr{A}} \otimes \mathbf{Z}_{2}: \Omega_{*}^{\text {Spin }} \otimes \mathbf{Z}_{2} \rightarrow \mathrm{KO}_{*}(\text { pt. }) \otimes \mathbf{Z}_{2}
$$

be represented by a spin manifold with positive scalar curvature?

Our Theorem implies that if this problem is solved affirmatively, then the original conjecture is true.

The author would like to express his hearty gratitude to Professor Aiko Hattori for his valuable suggestions and continuous encouragement.

We have learned since writing this paper that Jonathan Rosenberg has independently proved a parallel result except that he needs a larger power of 2 in dimensions congruent to $4 \bmod 8$.

Proof of the Theorem. In order to represent each class of $l_{k} \operatorname{ker} \hat{\mathscr{A}}_{k}$ by a spin manifold of positive scalar curvature, we need three types of manifolds. $P(E(M))$ denotes the complex or quaternion projective space bundle of the complex or quaternion vector bundle $E$ over the manifold $M . c \theta^{k}\left(\right.$ resp. $\left.h \theta^{k}\right)$ denotes a trivial $k$-dimensional complex (resp. quaternion) vector bundle. 
First, we consider a $4 k$-dimensional spin manifold $(k \geqslant 2)$

$$
X_{k}=P\left(h \lambda \oplus h \boldsymbol{\theta}^{2}\left(\mathbf{H} \mathbf{P}^{k-2}\right)\right),
$$

where $h \lambda$ is the canonical line bundle. By some calculation (cf. [9]), we get

$$
s_{(k)}(\mathscr{P}(\tau))\left[X_{k}\right]= \pm 2(2 k+1)(k-1) .
$$

Secondly, we consider a $4 k$-dimensional manifold $(k \geqslant 2)$

$$
P\left(\zeta \oplus c \boldsymbol{\theta}^{3}\left(P\left(\xi \oplus c \boldsymbol{\theta}^{1}\left(\mathbf{C P}^{2 k-4}\right)\right)\right)\right),
$$

where $\zeta$ and $\xi$ are complex line bundles. Let $a$ and $b$ be the first Chern classes of the canonical line bundles over $\mathbf{C} \mathbf{P}^{2 k-4}$ and $P\left(\xi \oplus c \boldsymbol{\theta}^{1}\left(\mathbf{C P}^{2 k-4}\right)\right)$ respectively, and let $\xi$ and $\zeta$ have the first Chern classes $l a$ and $m a+n b(l, m, n \in \mathbf{Z})$ respectively. For given numbers $l, m, n$, we denote the above manifold by $Y_{k, l, m, n}$. Then we get (cf. [3])

$$
s_{(k)}(\mathscr{P}(\tau))\left[Y_{k, l, m, n}\right]= \pm(2 k+1)(k-2)\left((m+n l)^{2 k-3}-m^{2 k-3}\right) / l .
$$

The necessary and sufficient condition for $Y_{k, l, m, n}$ to be spin is that $l+m$ is odd and $n$ is even.

Thirdly, we consider the $4 k$-dimensional manifold $H_{m, n, p, q}$, where $m+n-1=$ $2 k, n, m \geqslant 2$. $H_{m, n, p, q}$ is the hypersurface of $\mathbf{C P}^{m} \times \mathbf{C P}^{n}$ which is dual to $p u+q v$, where $u$ and $v$ are the first Chern classes of the canonical line bundles over $\mathbf{C P}^{m}$ and $\mathbf{C P}^{n}$ respectively, and $p$ and $q$ are integers. We get (cf. [10])

$$
s_{(k)}(\mathscr{P}(\tau))\left[H_{m, n, p, q}\right]= \pm\left(\begin{array}{c}
2 k+1 \\
m
\end{array}\right) p^{m} q^{n} .
$$

Since $m+n$ is odd, we may assume that $m$ is odd and $n$ is even. Then the necessary and sufficient condition for $H_{m, n, p, q}$ to be spin is that $p$ is even and $q$ is odd.

LEMMA. (1) $X_{k}(k \geqslant 2)$ and $Y_{k, l, m, n}(k \geqslant 2)$ carry metrics of positive scalar curvature.

(2) $H_{m, n, p, q}$ carries a metric of positive scalar curvature if $m<n$ and $p<0, q=-1$.

Proof. (1) $X_{k}$ and $Y_{k, l, m, n}$ carry such metrics because, for each complex or quaternion projective space bundle of a vector bundle, a metric of positive scalar curvature is constructed as follows. In the direction along the fibers, it is constructed from the ordinary metric of a complex or quaternion projective space and in the direction vertical to the fibers, it is constructed from an arbitrary metric of the base space using a metric connection of the vector bundle. This defines a Riemannian submersion with totally geodesic fibers. Shrinking the metric in the fibers, it deforms to a metric of positive scalar curvature (cf. [8]).

(2) We use a homogeneous coordinate $\left(\left[z_{0}, \ldots, z_{m}\right],\left[w_{0}, \ldots, w_{n}\right]\right)$ of $\mathbf{C P}^{m} \times \mathbf{C P}^{n}$. It is straightforward to see $H_{m, n, p, q}(m<n, p<0, q=-1)$ is represented by the nonsingular hypersurface of degree $(p, 1)$ defined by $\sum_{i=0}^{m} z_{i}^{p} w_{i}=0$ (cf. [10, p. 81, 131]). $H_{m, n, p, q}$ is a $\mathbf{C P}^{n-1}$-bundle over $\mathbf{C P}^{m}$ whose projection is inherited from the first projection $\mathbf{C P} m \times \mathbf{C P}^{n} \rightarrow \mathbf{C P}^{m}$. Give $H_{m, n, p, q}$ the metric induced from $\mathbf{C P}^{m} \times$ $\mathbf{C P}{ }^{n}$. Then pull back the metric of the base space $\mathbf{C P}^{m}$ in the direction vertical to the fibers. This defines a Riemannian submersion with totally geodesic fibers. Shrinking the metric in the fibers, one has the desired metric. 
Proposition. For each integer $k \geqslant 2$, there is a $4 k$-dimensional spin manifold $M_{k}$ which admits a metric of positive scalar curvature and

$$
s_{(k)}(\mathscr{P}(\tau))\left[M_{k}\right]= \begin{cases}2 & \text { if } 2 k+1 \neq r^{s} \text { for any prime } r \text { and integer } s, \\ 2 r & \text { if } 2 k+1=r^{s} \text { for some prime } r \text { and integer } s .\end{cases}
$$

Proof. The $s$-numbers of $X_{k}, Y_{k, 1,0,2}$ and $Y_{k, 0,1,2}$ are $\pm 2(2 k+1)(k-1)$, $\pm 2^{2 k-3}(2 k+1)(k-2)$ and $\pm 2(2 k+1)(k-2)(2 k-3)$ respectively. Taking the greatest common divisor of these three numbers, one can construct a spin manifold $N_{k}$ with its $s$-number $2(2 k+1)$.

Let $r$ be the odd prime that divides $2 k+1$. If $2 k+1$ is not a power of $r$, then one may write $2 k+1=r^{t} d$ with $d \not \equiv 0 \bmod r$. Then the $s$-number of $H_{r^{t}, r^{t}(d-1),-2,-1}$ is

$$
\pm\left(\begin{array}{c}
r^{t} d \\
r^{t}
\end{array}\right) 2^{r^{t}}
$$

and this is not congruent to $0 \bmod r$. If $2 k+1=r^{s}$ for some $s \geqslant 2$, then the $s$-number of $H_{r^{s-1}, r^{2}-r^{s-1},-2,-1}$ is

$$
\pm\left(\begin{array}{c}
r^{s} \\
r^{s-1}
\end{array}\right) 2^{r^{s-1}}
$$

and this is not congruent to $0 \bmod r^{2}$. Again taking the greatest common divisor, we construct $M_{k}$.

Now we can prove the Theorem. Since all torsion in the spin cobordism ring has order two, we restrict ourselves to $\Omega_{*}^{\text {Spin }}$ /Tor. Let $B^{\text {SO }} \subset \Omega_{*}^{\text {SO }} /$ Tor be the polynomial algebra generated by the classes $y_{i}(i \geqslant 1)$ characterized by

$$
\begin{aligned}
s_{(i)}(\mathscr{P}(\tau))\left[y_{i}\right] & = \pm m_{2 i} m_{2 i-1} \quad \text { for } i \geqslant 2, \\
s_{(1)}(\mathscr{P}(\tau))\left[y_{1}\right] & = \pm 2^{3} 3
\end{aligned}
$$

where $m_{i}=r$ if $i+1=r^{s}$ for some prime $r$ and positive integer $s$ and $m_{i}=1$ otherwise (cf. [10, p. 280]). Let $M_{k}$ be the manifold given in the Proposition. Since the $s$-number of $M_{k}$ is $2 m_{2 k}$, we may assume $M_{i}=y_{i}$ if $i=2^{s}$ and $M_{i}=2 y_{i}$ if $i \neq 2^{s}$ $(i \geqslant 2)$. Therefore $2 y_{i}(i \geqslant 2)$ is represented by a $4 k$-dimensional spin manifold with positive scalar curvature.

Note that $\Omega_{8 i}^{\text {Spin }} /$ Tor $=B_{8 i}$ and $\Omega_{8 i+4}^{\text {Spin }} /$ Tor $=2 B_{8 i+4}$ (cf. [10, p. 340]). Additive generators of $\Omega_{8 i+4}^{\mathrm{Spin}} /$ Tor are grouped into two types:

(i) $2 y_{1}^{2 i+1}$. The $\hat{A}$-genus is not zero and this cannot carry positive scalar curvature.

(ii) $2 y_{i_{1}} \cdots y_{i_{1}}, i_{1}+\cdots+i_{j}=2 i+1$ and $i_{j} \neq 1$. $2\left(2 y_{i_{1}} \cdots y_{i_{1}}\right)$ is spin cobordant to $\left(2 y_{i_{1}} \cdots y_{i_{j-1}}\right)\left(2 y_{i_{j}}\right)$, which is represented by a product manifold of a spin manifold and a spin manifold of positive scalar curvature. Similarly, generators of $\Omega_{8 i}^{\text {Spin }}$ are grouped into two types.

(i) $y_{1}^{2 i}$. This cannot carry positive scalar curvature.

(ii) $y_{i_{1}} \cdots y_{i_{1}}, i_{1}+\cdots+i_{j}=2 i$ and $i_{j} \neq 1.4 y_{i_{1}} \cdots y_{i}$ is spin cobordant to $\left(2 y_{i_{1}} \cdots y_{i_{j-1}}\right)\left(2 y_{i_{1}}\right)$ and carries positive scalar curvature.

The Theorem is the immediate consequence of these.

Proof of the Corollary. By the Theorem, $4 M$ carries positive scalar curvature. Then take the greatest common divisor. 


\section{REFERENCES}

1. D. W. Anderson, E. H. Brown, Jr. and F. P. Peterson, The structure of the spin cobordism ring, Ann. of Math. (2) 83 (1966), 47-53.

2. L. B. Bergery, La coubure scalaire des variétés riemanniennes, (Séminaire Bourbaki, 32e année, 1979/80, no. 556), Lecture Notes in Math., vol. 842, Springer-Verlag, Berlin and New York, pp. 225-245.

3. A. Borel and F. Hirzebruch, Characteristic classes and homogeneous spaces. I, Amer. J. Math. 8 (1958), 458-538.

4. M. Gromov and H. B. Lawson, Jr., The classification of simply connected manifolds of positive scalar curcature, Ann. of Math. (2) 111 (1980), 423-434.

5. N. Hitchin, Harmonic spinours, Adv. in Math. 14 (1974), 1-55.

6. A. Lichnerowicz, Spineurs harmoniques, C. R. Acad. Sci. Paris Sér. I Math. 257 (1963), 7-9.

7. J. Milnor, Remarks concerning spin manifolds, Differential Geometry and Combinatorial Topology, Princeton Univ. Press, Princeton, N. J., 1965, pp. 55-62.

8. B. O'Neill, The fundamental equations of a submersion, Michigan Math. J. 13 (1966), 459-469.

9. R. H. Szczarba, On tangent bundles of fiber spaces and quotient spaces, Amer. J. Math. 86 (1964), 685-697.

10. R. Stong, Notes on cobordism theory, Princeton Univ. Press, Princeton, N. J., 1968.

Department of Mathematics, Faculty of Science, University of Tokyo, Tokyo, Japan 